\title{
Electromagnetic Steering of Nanomedicine to the Olfactory Mucosa for Nose-to-Brain Drug Delivery
}

ISSN: 2637-7748

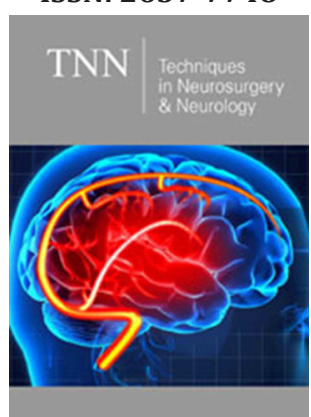

*Corresponding author: Jinxiang $\mathrm{Xi}$, Associate Professor, Department of Biomedical Engineering, USA

Submission: 泟 December 21, 2019

Published: 酮January 13, 2020

Volume 3 - Issue 2

How to cite this article: Jinxiang $\mathrm{X}$, Xiuhua April S. Electromagnetic Steering of Nanomedicine to the Olfactory Mucosa for Nose-to-Brain Drug Delivery. Tech Neurosurg Neurol.3(2). TNN.000556.2020.

DOI: 10.31031/TNN.2020.03.000556

Copyright@ Jinxiang X, This article is distributed under the terms of the Creative Commons Attribution 4.0 International License, which permits unrestricted use and redistribution provided that the original author and source are credited.

\author{
Jinxiang $\mathrm{Xi}^{1}$ and Xiuhua April $\mathrm{Si}^{2}$ \\ ${ }^{1}$ Associate Professor, Department of Biomedical Engineering, USA \\ ${ }^{2}$ Professor, Department of Aerospace, Industrial, and Mechanical Engineering, USA
}

\begin{abstract}
The nasal passage is not only very narrow but also with a complex structure, which filters most nasally inhaled aerosols, preventing effective nose-to-brain drug delivery with a conventional method. Different methods have been tested to optimize targeted drug delivery to the olfactory mucosa, where deposited drugs can enter the brain. These include pointed drug release, bi-directional intranasal delivery, electromagnetic guidance, and pulsating flows. Both in vitro experiments and computational simulations were used to quantify the aerosol deposition rate to the olfactory region. Results showed that point drug release and the bi-directional technique both yielded improved but very small percentage of deposition in the olfactory region. Electric charge or magnetize aerosol particles can further improve olfactory targeting by applying an appropriate external electric or magnetic field in the nose. The olfactory deposition was sensitive to the voltage of the electrode close to the nose. For both the normal and bi-directional deliveries, electric field guidance resulted in a significant increase ( $3 \sim 5$ times) in the olfactory deposition.
\end{abstract}

Keywords: Olfactory targeting; Personalized medicine; Electromagnetic steering; Nose-to-brain drug delivery

\section{Introduction}

Delivering drugs directly to the olfactory region has many advantages over traditional treatment of neurological disorders [1,2]. Extremely low efficiency of olfactory delivery using conventional nasal devices has discouraged clinical applications of direct nose-to-brain drug delivery and forestalled the development of newly engineered neurological medications devised for the nasal route of administration [3]. The poor drug delivery to the olfactory region is due to the following two factors: (1) the convoluted nasal passage, which blocks most of the inhaled aerosols to reach the olfactory region [4], and (2) no control over particle trajectories in the nose. New delivery methods have been explored to increase the delivery efficiency to the olfactory region. A catheter was Wang et al. [5] suggested to let a catheter slide beneath the olfactory region to administer medications. However, this protocol has the risk to irrigate the turbinate mucosa when inserting the catheter into the nasal passages. Gizurarson [6] evaluated the performance of a nasal spray with a small plume angle so that aerosols can reach the olfactory region. This technique requires a high spray speed or with a high pressure to overcome the flow resistance. Hoekman \& Ho [7] reported that a swirling flow in the nasal sprays resulted in improved olfactory dosages in rats than the non-swirling flow. Data extrapolation from rat to human, however, might not be comparable due to the vast inter-species discrepancies $[8,9]$. One such difference is the area of the olfactory epithelium, which covers $45 \%$ of the nasal surface in rats, but only $5.2 \%$ in human [10]. Considering that the possible deposition location of a particle largely depends on its initial position (i.e., the positioning of nozzle), Si et al. [11] released aerosols into a specific point of the nostril and demonstrated increased olfactory dosage than the method that aerosols sprayed into the entire nostril. However, the overall efficiency is still too low for clinical application. Examining all trails above, it is noted that after the aerosols enter the nose, their trajectories are solely determined by drag and inertia. Without external control, most of these aerosols will be blocked out by the labyrinthine nasal passages. This following section reviews the experimental and 
computational studies our laboratory performed in order to find more efficient and feasible targeted drug delivery methods to the olfactory region and paranasal sinuses. These included point drug release, electric guidance, magnetic control, and pulsating aerosols, individually and combined.

\section{Methods}

A hollow nose cast with a constant wall thickness was developed with an image-based nasal airway model [12]. The cast was separated into multiple sections to quantify sub-regional deposition fractions (Figure 1). Step-shaped grooves were built at the ends of each section for sealing and assembly purpose $[13,14]$. The efficiency of olfactory delivery for different drug release positions, with or without electric and magnetic fields, and pulsating flow were studied [15]. For in vitro tests of point drug release (i.e., releasing particles into a small point within the nostril as opposed to the entire nostril), nozzles or adaptors were used to focus the nebulizer output. Three types of nebulizers, vibrating mesh, ultrasound, and jet [16] were studied. In the in vitro tests of electric-guided delivery, a re-engineered powder coating system (Nolensville, TN) was utilized to charge the dry powder. A DC power supply charged insulated copper plates were used to generate electric fields. Two nasal delivery methods were considered, one being the normal breathing (unidirectional) and the other being bidirectional $[17,18]$. An electronic scale (Sartorius) with a precision of $0.01 \mathrm{mg}$ was used to measure the aerosol mass. Sar-Gel was used to visualize the local deposition of the nasal sprays and nebulized droplets [19-21].

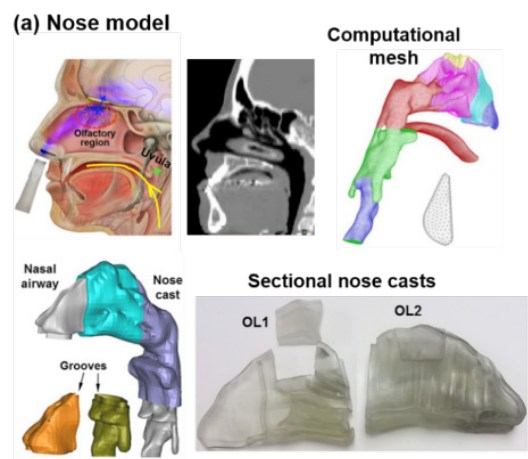

(b) Two delivery methods
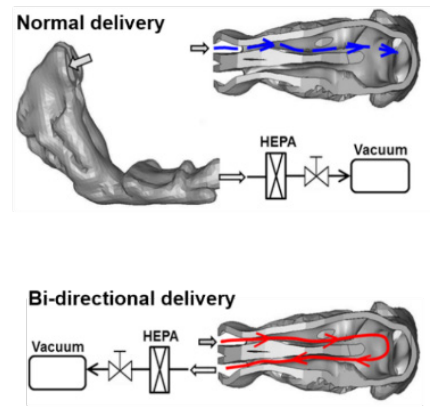

Figure 1: Nose model and experimental methods: (a) computational and in vitro nasal airway models, and (b) normal and bi-directional delivery techniques.

For computational modeling, particle motions were tracked using the Lagrangian approach in Comsol:

$$
\begin{aligned}
& \frac{d\left(m_{p} v_{i}\right)}{d t}=\frac{f}{\tau_{p} C_{c}} m_{p}\left(u_{i}-v_{i}\right)+F_{i, L i f t} . \\
& +F_{i, \text { Electrophoretic }}+F_{i, \text { Magnetophoretic }}+F_{i, \text { Acoutophoretic }}
\end{aligned}
$$

where $u_{i}$ and $v_{i}$ are velocities of the airflow and particle, respectively, and $f$ is the drag coefficient [22]. The particle motion is a function of all external forces applied on the particle, which includes drag force, Saphmann lift force, and electro-, magneto-, and acousto-phoretic forces. The electrophoretic force is calculated as $f_{i \text {,Electrophoretic }}=n e E$, where $n$ is the particle charge number, $e$ is the elementary charge, and $\mathrm{E}$ is the intensity of the electric field [23]. The magnetophoretic force is calculated as $F_{i, \text { Magnetophoretic }}=\left(\mu_{0} m_{e f f} \cdot \nabla\right) H$ , where $\mathrm{H}$ is magnetic intensity, and $\mu_{f}$ and $\mu_{p}$ are the magnetic permeability of the fluid and particle $[24,25] . m_{\text {eff }}$ is the induced magnetization and proportional to $d_{p}^{3}$. The acoustophoretic force is proportional to $-\nabla\left(d_{p}^{3} P^{2}\right)$ [26]. More details of model reconstruction and numerical methods using Comsol can be seen in a recent video article from our lab [23].

\section{Results}

\section{Electric guidance}

The inhalation device with electric guidance consists two components: an aerosol generation/charging module and a headmounted brace [27]. Electrodes are embedded in the nasal mask, which is anchored by the brace relative to the patient nose (Figure 2a). One conceptual design of the aerosol generation/charging device is depicted in Figure $2 \mathrm{~b}$ with a nebulizer, an electric charger, and a nozzle for point-release. Figure $2 b$ shows a prototype manufactured by a 3D printer (Object30 Pro). Desirable parameters for such a design should include small particle size $(<4 \mu \mathrm{m})$, low exiting velocity, and high electric charge for optimal electric guidance [28-30]. Numerical modeling of olfactory deposition was first conducted for scenarios of point- or entire-nostril release with and without electric guidance. With a proper electric field, $3.7 \%$ of aerosols released from the entire nostril deposited in the olfactory region, in contrast to $0.06 \%$ in the absence of electric guidance (Figure 2c). Point release further enhanced olfactory delivery by two orders of magnitude. The majority ( $90 \%$ in the optimal scenario) of inhaled aerosols were bypassed the valve and turbinate and guided to the olfactory region (red ellipse) (Figure 2c). Figure 2d compares experimental particle deposition patterns without and with electric charges using powder. Similarly, most particles were trapped in the anterior nose without electric guidance and only very few particles reached the olfactory region. However, Electric guidance significantly improves olfactory delivery (blue dashed ellipse, Figure 2d) with a 5.2-fold enhancement for normal (unidirectional) breathing and a 3.0-fold enhancement for bi-directional (Figure 2d). 

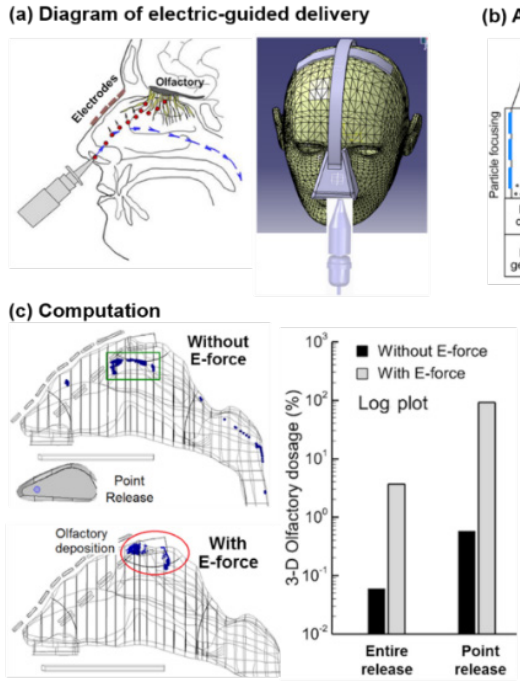

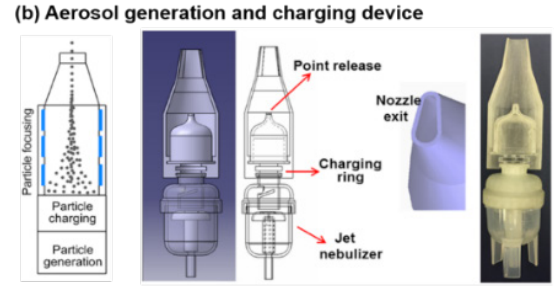

(d) In vitro tests

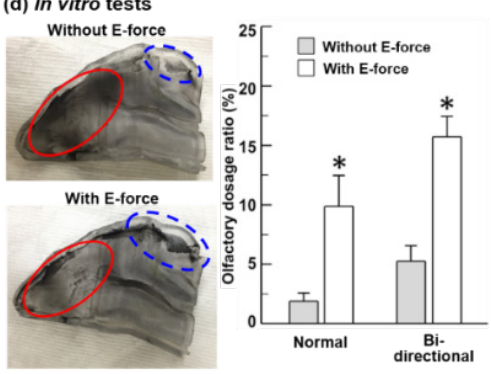

Figure 2: Targeted olfactory delivery with electric guidance: (a) diagram of electric-guided delivery, (b) aerosol generation and charging device, (c) computed olfactory deposition with/without electric guidance, and (d) in vitro experiments with/without electric guidance.

\section{Magnetic guidance}

The feasibility of magnetic particle guidance was first evaluated in a two-plate channel (H: $10 \mathrm{~mm}, \mathrm{~L}: 150 \mathrm{~mm})$ with permanent magnets on the outside edges [31]. Particles are composed of drug and iron nanoparticles with a relative magnetic permeability of 700 , density $1500 \mathrm{~kg} / \mathrm{m}^{3}$, and a particle size of $15 \mu \mathrm{m}$. In the absence of a magnetic force, particles follow parabolic paths due to gravity (Figure 3a). By varying the strength of the magnetic field, an appropriate intensity was found which can achieve an effective control of the particle motion. For instance, guiding particles to move horizontally instead of settling by counteracting the gravity (layout 1) or targeting particles to a specific site by applying a strong attraction force (layout 2). It is noted that the resultant magnet field was non-uniform, being stronger near the magnet bars but quickly decreasing in strength away from them. To test the magnetic control in olfactory targeting, magnet bars are put outside the nasal passage to produce a nonuniform magnetic field
(Figure 3b). With the point release and an appropriate magnetic field, a deposition fraction of $45 \%$ was predicted (Figure $3 b$ \& $3 c)$. In contrast, only a small fraction (1.2\%) of particles was predicted to deposit in the olfactory region when released from the entire nostril, even with an optimal magnetic field (Figure $3 \mathrm{~b}$ ). The effectiveness of magnetic control also highly depends on the particle sizes. As shown in Figure 3c above, particles only sized between $10-20 \mu \mathrm{m}$ can be effectively guided for olfactory targeting. Insignificant olfactory dosing occurs when the particle diameter is smaller than $10 \mu \mathrm{m}$ or larger than $20 \mu \mathrm{m}$. Particles smaller than $10 \mu \mathrm{m}$ have negligible responsiveness to the magnetic field, while particles larger than $20 \mu \mathrm{m}$ will be lost in the anterior nose due to high inertia. It is reminded that the magnetic force on a particle increases with the third power of the particle diameter $\left(d_{p}{ }^{3}\right)$, as opposed to the first power of the magnetic field strength. In this study, the optimal olfactory deposition is achieved for particles in $13-17 \mu \mathrm{m}[31,32]$.
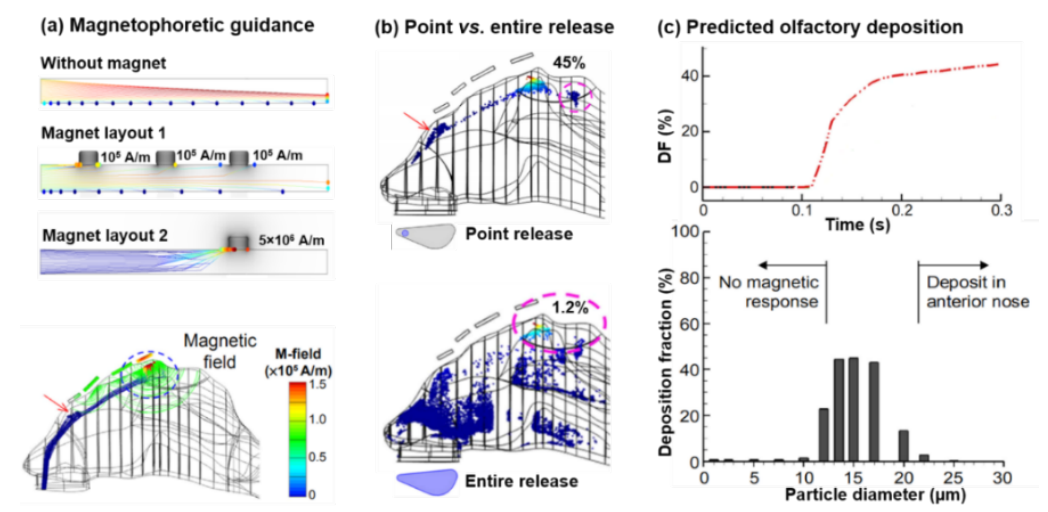

Figure 3: Targeted olfactory delivery with magnetic guidance: (a) magnetophoretic guidance in a two-plate channel, (b) comparison of particle deposition patterns between point release and entire-nostril release, and (c) numerically predicted olfactory deposition. 


\section{Discussion}

In this paper, we reviewed a series of attempts of our lab to enhance targeted olfactory drug delivery region. Both experimental and numerical tests were performed to this objective by evaluating different mechanisms of particle control in the nose. Improved olfactory dosages were observed with strategies of drug point release, electric, and magnetic guidance, but at varying levels. It is practical to attain clinically significant doses by using a combination of the point-release technique and the electric-guidance of charged particles. In comparison to conventional inhalation devices, the drug point-release approach significantly reduced the drug losses in the valve and turbinate region. However, even at its optimal delivery efficiency $(\sim 6.7 \%)$, the point-release technique alone still falls short of the needs of the direct nose-to-brain drug delivery with effective outcomes and no noticeable adverse side-effects. Active controls of particle motion with an electromagnetic or acoustic field have been numerically demonstrated to dispense enhanced doses to the targeted sites. Complimentary in vitro tests also demonstrated the potential of electric guidance; an olfactory delivery efficiency of $16 \%$ was measured with charged particles and bi-directional nasal delivery. Future studies are needed to consider the intra-subject and inter-subject variability in olfactory delivery efficiencies $[33,34]$.

\section{References}

1. Hanson L, Frey W (2007) Strategies for intranasal delivery of therapeutics for the prevention and treatment of neuro AIDS. J Neuroimmune Pharmacol 2(1): 81-86.

2. Mistry A, Stolnik S, Illum L (2009) Nanoparticles for direct nose-to-brain delivery of drugs. Int J Pharm 379(1): 146-157.

3. Illum L (2002) Nasal drug delivery: New developments and strategies. Drug Discov Today 7 (23): 1184-1189.

4. Xi J, Longest PW (2009) Characterization of sub-micrometer aerosol deposition in extra thoracic airways during nasal exhalation. Aerosol Sci Technol 43(8): 808-827.

5. Wang J, Bentz J, Anderson R (2007) Nasal device for delivery to the olfactory region. US $20070119451 \mathrm{~A} 1$.

6. Gizurarson S (2003) Method for administration of active substances to the olfactory region. Patents CA 2298596.

7. Hoekman JD, Ho RJY (2011) Effects of localized hydrophilic mannitol and hydrophobic nelfinavir administration targeted to olfactory epithelium on brain distribution. AAPS Pharmscitech 12(2): 534-543.

8. Corley RA, Kabilan S, Kuprat AP, Carson JP, Minard KR, et al. (2012) Comparative computational modeling of airflows and vapor dosimetry in the respiratory tracts of rat, monkey, and human. Toxicol Sci 128(2): 500-516.

9. Xi J, Si XA, Kim J, Zhang Y, Jacob RE, et al. (2016) Anatomical details of the rabbit nasal passages and their implications in breathing, air conditioning, and olfaction. The Anatomical Record 299(7): 853-868.

10. Xi J, Kim J, Si XA, Corley RA, Zhou Y (2015) Modeling of inertial depositions in scaled models of rat and human nasal airways: towards in vitro regional dosimetry in small animals. J Aerosol Sci 99: 78-93.

11. Si X, Xi J, Kim J, Zhou Y, Zhong H (2013) Modeling of release position and ventilation effects on olfactory aerosol drug delivery. Respir Physiol Neurobiol 186(1): 22-32.
12.Xi J, Berlinski A, Zhou Y, Greenberg B, Ou X (2012) Breathing resistance and ultrafine particle deposition in nasal-laryngeal airways of a newborn, an infant, a child, and an adult. Ann Biomed Eng 40(12): 2579-2595.

13. Zhou Y, Xi J, Simpson J, Irshad H, Cheng YS (2013) Aerosol deposition in a nasopharyngolaryngeal replica of a 5-year-old child. Aerosol Sci Technol 47(3): 275-282.

14. Zhou Y, Guo M, Xi J, Irshad H, Cheng YS (2014) Nasal deposition in infants and children. J Aerosol Med Pulm Drug Deliv 27(2):110-116.

15. Xi J, Si X, Longest W (2014) Electrostatic charge effects on pharmaceutical aerosol deposition in human nasal-laryngeal airways. Pharmaceutics 6(1): 26-35.

16. Xi J, Yuan JE, Zhang Y, Nevorski D, Wang Z, et al. (2016) Visualization and quantification of nasal and olfactory deposition in a sectional adult nasal airway cast. Pharm Res 33(6): 1527-1541.

17.Xi J, Wang Z, Nevorski D, White T, Zhou Y (2017) Nasal and olfactory deposition with normal and bidirectional intranasal delivery techniques: in vitro tests and numerical simulations. J Aerosol Med Pulm Drug Deliv 30(2): 118-131.

18. Djupesland PG, Messina JC, Mahmoud RA (2013) Breath powered nasal delivery: a new route to rapid headache relief. Headache 53(2): 72-84.

19. Kundoor V, Dalby RN (2010) Assessment of nasal spray deposition pattern in a silicone human nose model using a color-based method. Pharm Res 27(1): 30-36.

20. Kundoor V, Dalby RN (2011) Effect of formulation- and administrationrelated variables on deposition pattern of nasal spray pumps evaluated using a nasal cast. Pharm Res 28(8): 1895-1904.

21. Xi J, Yang T, Talaat K, Wen T, Zhang Y, et al. (2017) Visualization of local deposition of nebulized aerosols in a human upper respiratory tract model. J Vis 1-13.

22. Morsi SA, Alexander AJ (1972) An investigation of particle trajectories in two-phase flow systems. J Fluid Mech 55(2): 193-208.

23. Si XA, Xi J (2016) Modeling and simulations of olfactory drug delivery with passive and active controls of nasally inhaled pharmaceutical aerosols. J Vis Exp (111): e53902-e53902.

24. Gao Y, Jian YC, Zhang LF, Huang JP (2007) Magnetophoresis of nonmagnetic particles in ferrofluids. J Phys Chem C 111(29): 1078510791.

25. Ribeiro P, Si X (2012) Electric and magnetic circuit. In: Beaty HW, Fink DG, (Eds.), Standard handbook for electrical engineering. (16 ${ }^{\text {th }}$ edn), pp. $1-58$.

26. Xi J, Si XA, Peters S, Nevorski D, Wen T, et al. (2017) Understanding the mechanisms underlying pulsating aerosol delivery to the maxillary sinus: In vitro tests and computational simulations. Int J Pharm 520(1): 254-266.

27. Xi J, Eddie Yuan J, Alshaiba M, Cheng D, Firlit Z, et al. (2016) Design and testing of electric-guided delivery of charged particles to the olfactory region: experimental and numerical studies. Curr Drug Deliv 13(2): 265274.

28. Xi J, Si X, Gaide R (2014) Electrophoretic particle guidance significantly enhances olfactory drug delivery: a feasibility study. PLoS ONE 9(1): e86593.

29. Xi J, Yuan JE, Si XA (2016) Simulation study of electric-guided delivery of $0.4 \mu \mathrm{m}$ monodisperse and polydisperse aerosols to the ostiomeatal complex. Comput Biol Med 72: 1-12.

30. Golshahi L, Longest PW, Holbrook L, Snead J, et al. (2015) Production of highly charged pharmaceutical aerosols using a new aerosol induction charger. Pharm Res 32(9): 3007-3017. 
31. Xi J, Zhang Z, Si X (2015) Improving intranasal delivery of neurological nanomedicine to the olfactory region using magnetophoretic guidance of microsphere carriers. Int J Nanomedicine 10: 1211-1222.

32. Xi J, Si XA, Kim J, Zhang Y, Jacob RE, Kabilan S, et al. (2016) Anatomical details of the rabbit nasal passages and their implications in breathing, air conditioning, and olfaction. Anat Rec 299(7): 853-868.
33.Xi J, Yang T (2019) Variability in oropharyngeal airflow and aerosol deposition due to changing tongue positions. J Drug Deliv Sci Technol 49: 674-682.

34. Lu J, Xi J, Langenderfer JE (2017) Sensitivity analysis and uncertainty quantification in pulmonary drug delivery of orally inhaled pharmaceuticals. J Pharm Sci 106(11): 3303-3315. 